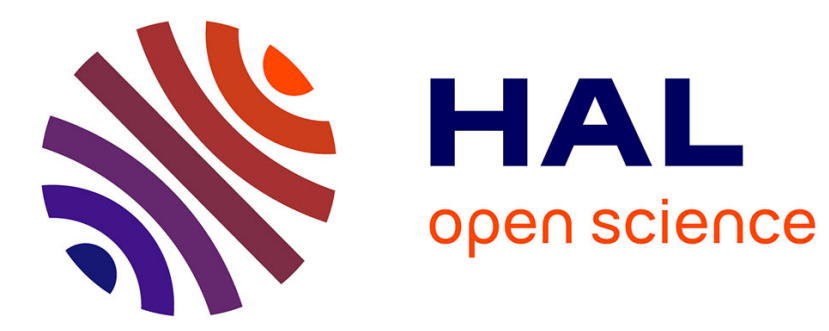

\title{
Applications of femtosecond Bessel beams to laser ablation
}

François Courvoisier, Jie Zhang, Manoj Bhuyan, Maxime Jacquot, John Michael Dudley

\section{- To cite this version:}

François Courvoisier, Jie Zhang, Manoj Bhuyan, Maxime Jacquot, John Michael Dudley. Applications of femtosecond Bessel beams to laser ablation. Applied physics. A, Materials science \& processing, 2013, 112, pp.29 - 34. 10.1007/s00339-012-7201-2 . hal-00845925

\section{HAL Id: hal-00845925 \\ https://hal.science/hal-00845925}

Submitted on 18 Jul 2013

HAL is a multi-disciplinary open access archive for the deposit and dissemination of scientific research documents, whether they are published or not. The documents may come from teaching and research institutions in France or abroad, or from public or private research centers.
L'archive ouverte pluridisciplinaire HAL, est destinée au dépôt et à la diffusion de documents scientifiques de niveau recherche, publiés ou non, émanant des établissements d'enseignement et de recherche français ou étrangers, des laboratoires publics ou privés. 


\title{
Applications of femtosecond Bessel beams to laser ablation
}

\author{
F. Courvoisier, J. Zhang, M. K. Bhuyan, M. Jacquot, J.M. Dudley \\ Département d'Optique P.M. Duffieux, Institut FEMTO-ST, UMR 6174 CNRS Université de Franche-Comté, 25030 Besançon \\ Cedex, France
}

Received: date / Revised version: date

\begin{abstract}
Nondiffracting Bessel beams have been recently applied to single shot ultrafast laser processing of high aspect ratio nanochannels. We review the principles and benefits of nondiffracting Bessel beams for material processing, and we compare experimental results with numerical simulations. We show how the stationarity of nonlinear propagation of ultrafast laser pulses allows high aspect ratio material processing.
\end{abstract}

\section{Introduction}

Nondiffracting beams such as Bessel beams beams exhibit a near constant intensity profile along the propagation [1]. Since the intensity maximum is strongly localized over a distance that exceeds the Rayleigh range of Gaussian beams by orders of magnitude, they have attracted a tremendous interest in both linear and nonlinear optics. These beams have been successfully used in the field of all-optical manipulation, and their unique properties of self-reconstruction and stability under nonlinear propagation in dielectrics have made them ideal candidates for highly localized and controlled energy deposition in transparent materials [2-6].

Our motivation here is to show how they can be applied to the field of femtosecond laser processing [7-9], with a particular interest in the fabrication of high aspect ratio channels for micro/nanofluidics and $2 \mathrm{D}$ photonic crystals. Indeed, deep nanochannel processing is at present a key technological issue for these important application fields and the existing technologies for material nanoprocessing such as FIB milling or DRIE are not well suited to high aspect ratio (>10:1) nanoprocessing. However, although high aspect ratio material processing with Bessel beams has already been experimentally demonstrated, numerical simulations at ablation-level intensities have not been reported to our knowledge. Here,

Send offprint requests to: francois.courvoisier@femto-st.fr we compare experimental results with standard optical nonlinear propagation simulations. Specifically, we show how stationarity of the nonlinear propagation depends on the specific ionization processes. We also demonstrate that the robustness of Bessel beams allows the point by point drilling of a periodic lattice of nanochannels without modifying the size of the Bessel central core.

\section{Nondiffracting Bessel beams}

Bessel beams are solutions to the paraxial wave equation with a propagation invariant profile. However, the propagation invariance property can be maintained over infinite distances only for infinite energy beams. In practice, a good approximation is given by Bessel-Gauss beams [10].For simplicity here, zero-order Bessel-Gauss beams beams will be referred to as Bessel beams.

Bessel beams can be viewed as a rotationally symmetric interference of plane waves whose wavevectors lie on a cone. Figure 1 shows the intensity profile of a Bessel beam : a high intensity central core is surrounded by several circular lobes of lower intensity. A standard Bessel-Gauss beam is determined by two independant parameters: the conical angle, that is the angle $\theta$ made by the wavevectors with the optical axis, which determines the size of the lobes, and the waist of the initial Gaussian beam, that determines the length of the Bessel beam, as can be seen on figure 1 .

Many ways exist to produce Bessel beams, but all need to apply spatially a linear phase with rotational symmetry. We can divide Bessel beam generation in two broad families: one is the direct generation with an axicon lens or a spatial light modulator (SLM) [7,11], the second is based on Fourier-transforming an annular intensity distribution by use of a lens [12]. Obviously, since the first family is based on spatial phase shaping while the second is amplitude shaping, it is generally preferred to use axicons and SLM for femtosecond laser ablation. Spatial shaping with SLMs provides flexibility, and on- 
(a)

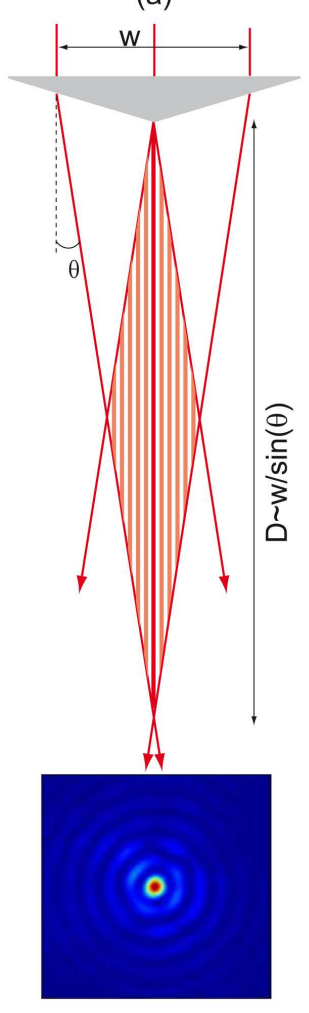

(b)

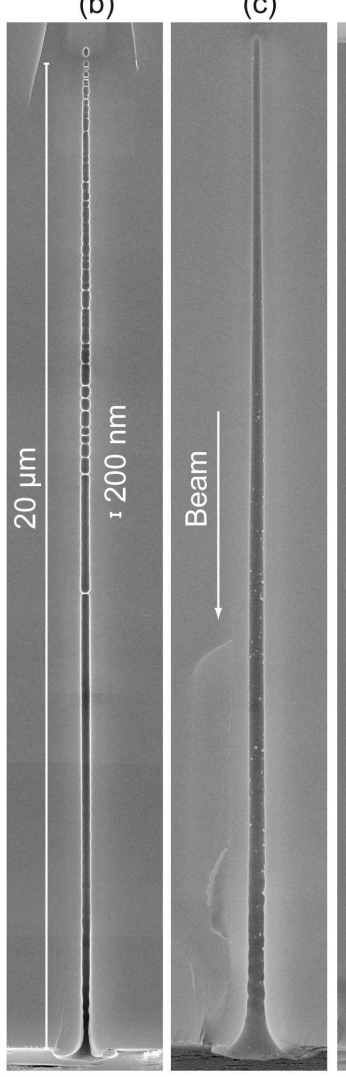

(d)

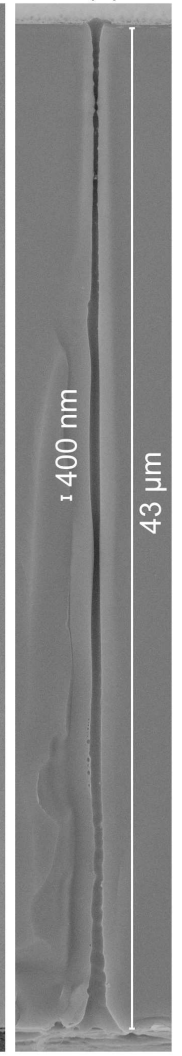

Fig. 1 (a) Geometrical structure of a Bessel beam of conical angle $\theta$ created by an axicon from a Gaussian beam of waist $w$. (inset) experimental cross section of such a beam. The central spot size is $0.7 \mu \mathrm{m}$ FWHM. (b)-(c) SEM images of nanochannels produced by single shot illumination at 0.65 and $0.85 \mu \mathrm{J}$ in glass. (d) SEM image of the channel produced by a single $3 \mu \mathrm{J}$ pulse through a $43 \mu \mathrm{m}$ thick glass slide.

axis intensity engineering has been demonstrated to produce Bessel beams with a constant intensity in the central core along the propagation [12].

Several properties of Bessel beams are advantageous to the field of laser ablation. Specifically, since the intensity is highly localized over a distance that exceeds the Rayleigh range by orders of magnitude, it is possible to realize surface processing on non-flat samples and remove the constraint of sample positioning towards the laser focus that is critical to realize nanometric damages $[7,13,14]$. Moreover, the self-healing property of nondiffracting beams allows avoidance of beam perturbation by obstructions or particles in the beam path.

We note that processing with Bessel beams has been performed on metals, but the linear absorption of light prevents the drilling of deep structures [15]. This approach is better suited to dielectrics $[8,9,16]$.

The strong dynamical interplay between light and matter during the propagation of ultrafast laser pulses at ablation-level intensity leads to a high degree of spatio- temporal distorsion of Gaussian shaped pulses [17], which renders the drilling of high-aspect ratio channels challenging [18-20]. In contrast, Bessel beams are resistant to the Kerr effect and nonlinear losses even at 100 critical powers [5]. This latter property has been numerically and experimentally tested for low plasma densities and small conical angles. Here we will numerically investigate the regime of high conical angles and plasma densities close to the critical densities for material damage and ablation.

\section{Single shot nanochannel processing with Bessel beams}

The experimental setup for micro and nano-channel processing with Bessel beam is described in details in references $[7,9]$. Briefly, individual femtosecond laser pulses from an amplified Ti:Sapphire source at $800 \mathrm{~nm}$ central wavelength are reflected on a spatial light modulator (SLM, Hamamatsu PAL-SLM) and shaped to a Bessel beam that is spatially filtered and demagnified by a factor 278. This setup realizes the exact demagnified image of the SLM on the image focal plane of a microscope objective, this image plays the role of a virtual axicon illuminated by a Gaussian beam with waist $\sim 10 \mu \mathrm{m}$. A micro-Bessel beam is then generated from the focal plane of a microscope objective with a conical angle of $25^{\circ}$ in air $\left(17^{\circ}\right.$ in glass), and the central lobe of the Bessel beam is $660 \mathrm{~nm}$ in diameter FWHM. In the study presented here, we used Corning 0211 glass samples with thickness $150 \mu \mathrm{m}$. The pulse duration was measured $\sim 230$ fs at the sample site.

The virtual axicon was imaged at a distance $D$ from the exit side of the samples, chosen so that the beam extends up to the exit side. After single shot illumination, the samples were cleaved, metallized and imaged with Scanning Electron Microscopy (SEM) without mechanical nor chemical cleaning. Figure 1(b) and (c) show the result for pulse energies of $0.65 \mu \mathrm{J}$ and $0.85 \mu \mathrm{J}$. Remarkably, the diameter of the nanochannels thus produced varies extremely slowly along the longitudinal dimension, and their aspect ratio was measured to be as high as 100:1. The uniformity of the diameter therefore suggests that the nonlinear propagation is close to invariant. In the following, we compare our experimental results to numerical simulations.

\section{Numerical model}

To simulate the nonlinear propagation of femtsecond pulses in air and glass, we used a standard model based on the nonlinear Schrödinger equation (NLSE) to model the evolution of the scalar electric field [5,21]:

$$
\frac{\partial E}{\partial z}=\frac{i}{2 k_{0}}\left(\frac{\partial^{2}}{\partial r^{2}}+\frac{1}{r} \frac{\partial}{\partial r}\right)-\frac{i k^{\prime \prime}}{2} \frac{\partial^{2} E}{\partial t^{2}}+\frac{i k_{0} n_{2}}{n_{0}}|E|^{2} E
$$


Table 1 Parameters used in the simulations. The coefficients were determined from reference [22].

\begin{tabular}{lll}
\hline & Parameter & Value \\
\hline$k^{\prime \prime}$ & Dispersion & $446 \mathrm{fs}^{2} / \mathrm{cm}$ \\
$\tau_{c}$ & Collision time & $1.28 \mathrm{fs}$ \\
$n_{0}$ & index of refraction & 1.53 \\
$n_{2}$ & Kerr index & $3.45 \times 10^{-20} \mathrm{~m}^{2} / \mathrm{W}$ \\
$U_{g}$ & Bandgap & $4.2 \mathrm{eV}$ \\
$\beta_{K}$ & Multiph. absorption & $4.7 \times 10^{-25} \mathrm{~cm}^{2 \mathrm{~K}-3} \mathrm{~W}^{1-\mathrm{K}}$ \\
$\sigma_{K}$ & Multiph. cross section & $7 \times 10^{-7} \mathrm{~cm}^{2 \mathrm{~K}-3} \mathrm{~s}^{-1} \mathrm{~W}^{-\mathrm{K}}$ \\
$K$ & Multiphoton order & 3 \\
$\tau_{r}$ & Recombination time & $150 \mathrm{fs}$ \\
$\rho_{0}$ & Total electron density & $2.1 \times 10^{-22} \mathrm{~cm}^{-3}$ \\
$t_{1 / 2}$ & Pulse duration & $230 \mathrm{fs}$ \\
$w_{0}$ & Gaussian waist & $10.1 \mu \mathrm{m}$ \\
\hline
\end{tabular}

$$
-\frac{\sigma}{2}\left(1+i \omega_{0} \tau_{c}\right) \rho E-\frac{\beta_{K}}{2}|E|^{2 K-2} E\left(1-\frac{\rho}{\rho_{0}}\right)
$$

where $t=t_{l a b}-z / v_{g}$ corresponds to the co-moving time and the material parameters are explicited in Table 1. The evolution of the free electron-hole plasma is governed by:

$$
\frac{\partial \rho}{\partial t}=\left(\frac{\sigma}{U_{g}} \rho|E|^{2}+\sigma_{k}|E|^{2 K}\right)\left(1-\frac{\rho}{\rho_{0}}\right)-\frac{\rho}{\tau_{r}}
$$

The electron Brehmsstrahlung cross-section is defined within the Drude model as $\sigma=k \omega_{0} \tau_{c} / n_{0}^{2} \rho_{c}\left(1+\omega_{0}^{2} \tau_{c}^{2}\right)$. In this numerical model, self-steepening was neglected, and we note that the main influence on the propagation is due to the contribution of plasma. Since most of the material constants are not tabulated for Corning 0211 glass, we have chosen the constants for BK7 since the bandgaps of these material are very close [23].

The initial pulse at the virtual axicon position $z=0$ is a pulsed Bessel beam [5]:

$$
E(z=0, r, t)=E_{0} e^{-r^{2} / w_{0}^{2}-2 \ln (2) t^{2} / t_{1 / 2}^{2}} e^{-i k_{0} r \sin (\theta) / n_{0}}
$$

This pulse shape was linearly back-propagated to $z=-70 \mu \mathrm{m}$ before being launched in the nonlinear propagation regime. The numerical integration is based on a split-step Fourier algorithm for equation (1) and fourth order Runge-Kutta algorithm for equation (2). Details of the modelling can be found in reference [24].

\section{Single shot Nanochannel processing with Bessel beams}

Figure 2 compares the distribution of the fluence in a plane containing the optical propagation axis $z$ and the transverse direction $r$ (a) in the purely linear regime to the maximal free-electron density distribution $\rho(r, z)$ for a $0.65 \mu \mathrm{J}, 230 \mathrm{fs}$ laser pulse with pure nonlinear losses (b) and with nonlinear losses and avalanche terms (c) in
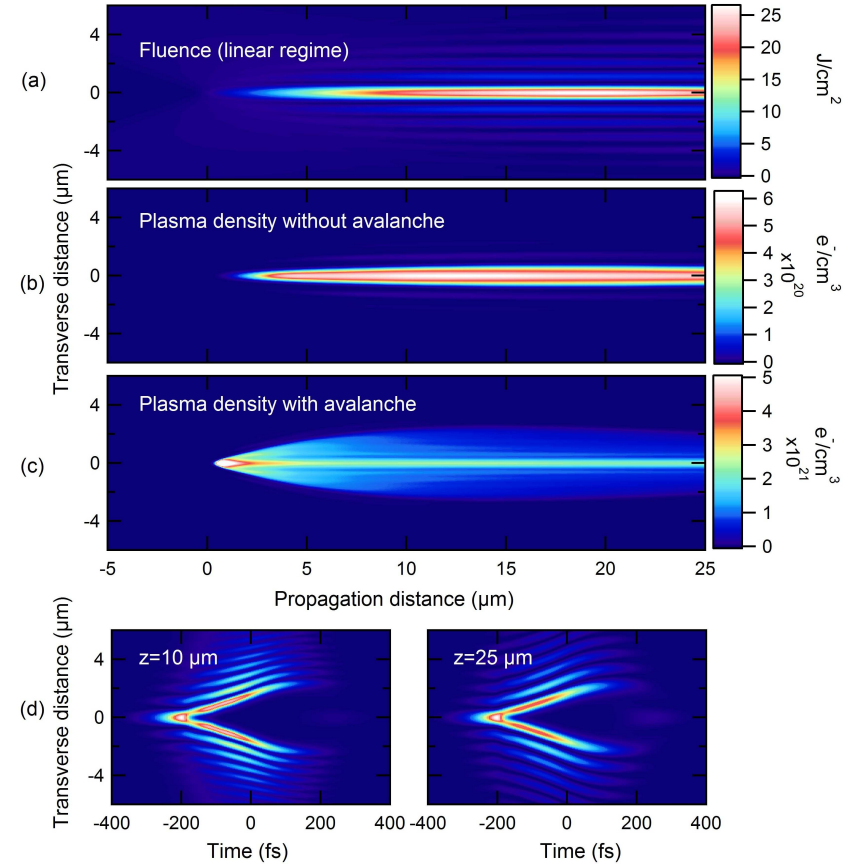

Fig. 2 Simulation of the propagation of a $0.65 \mu \mathrm{J}$ pulse with geometrical characteristics identical to the experimental ones (cf Fig.1 (b)). (a) Fluence distribution in the linear regime. (b) Free-electron plasma density produced without avalanche term (c) Free-electron plasma density produced with the full model. Note that the plasma density is one order of magnitude larger than in (b). (d) comparison of the spatio-temporal intensity distributions $|E(r, t)|^{2}$ at $z=5 \mu \mathrm{m}$ and $z=25 \mu \mathrm{m}$.

equation (2). Note that the order of magnitudes differ in the colorscale between (b) and (c): the plasma density with avalanche is ten times larger in the central region.

It is apparent that a dense $\left(>10^{20} \mathrm{e}^{-} / \mathrm{cm}^{3}\right)$ free electron plasma channel that is produced and the peak is confined within less than $1 \mu \mathrm{m}$ FWHM in the transverse direction. As predicted by the model of Porras et al [4], a model with pure nonlinear losses, without avalanche term, generates a plasma channel that is invariant in the longitudinal direction. The stationarity of the Bessel beam propagation arises from the conical structure of the beam. Indeed, as can be qualitatively understood from figure 1(a), the field amplitude contained in the outer lobes at a propagation distance $z$ contributes to the intensity in the central core at further propagation distance. The confinement of the plasma density distribution within the central lobe of the Bessel beam therefore leaves the outer lobes unaffected, allowing the buildup of a high intensity peak and plasma density in the central lobe at further propagation distance. We observe however that the avalanche term reduces the plasma density confinement through the generation of more free electron plasma out of the Bessel beam central core. In addition, we observe the effect of intensity clamping (not shown) when the energy per pulse is increased. Noticeably, the plasma channel can exceed the critical plasma density. 
This is attributed to the fact that the diameter of the plasma channel is smaller than the characteristic absorption length of the plasma at critical density $(\sim 1 \mu \mathrm{m})$.

We note that the length and invariance of the plasma channel are in qualitative agreement with our experimental results. However, our numerical results strongly depend on the exact value of the avalanche coefficient. Moreover, in our experimental results, no damage can be correlated to a high plasma density peak as the one that is generated close to the onset of the Bessel beam $(z=0)$, which indicates that the avalanche coefficient may be overestimated.

Another important characteristic of the propagation of non-diffracting Bessel beams is shown in Figure 2(d). It presents the intensity distribution in space-time coordinates $(r, t)$ at two different positions $z=10 \mu \mathrm{m}$ and $z=25 \mu \mathrm{m}$ for the full model including avalanche. We observe that there is no qualitative difference in the pulse shapes in the nonlinear propagation, showing a close-tostationarity propagation, even in presence of avalanche. In contrast, ultrafast pulses with a spatially Gaussian profile in similar conditions would exhibit spatio-temporal pulse splitting and a much more complex propagation dynamics $[17,25]$. Our results therefore qualitatively support the reason why the diameter of the channels drilled with Bessel beams is almost invariant along the channel length.

Figure 1(d) shows the image of a through channel drilled by a single laser shot through a $43 \mu \mathrm{m}$ thick glass membrane, which demonstrates that through channel drilling is possible when the threshold intensity is exceeded on both sides of the sample. However, we note that the channel diameter is less uniform along the structure, which could arise from interface effects. However, a close comparison to numerical simulations requires more accurate modelling of the free electron plasma generation.

\section{Variation of channel length and diameter}

Figure 3 recalls experimental results presented in reference [9]: we demonstrated that the diameter of the channels varies quasi-linearly with pulse energy, and that the length of the channels is linearly determined by the position of the virtual axicon in the sample.

We observe from our numerical results that the diameter over which the free electron plasma distribution exceeds $\sim 10^{21} \mathrm{~cm}^{-3}$ is larger than the diameter of the drilled channel. More, this diameter increases when the conical angle is smaller (looser focusing). In contrast, we experimentally observed that for a given pulse energy, the plasma channel diameter decreases when the conical angle was reduced, as shown in Figure 3(top). This shows, as expected, that the diameter of the channels is determined by the deposited energy density [9]

In addition, we can observe on Figure 2 that the plasma density is negligible for longitudinal positions
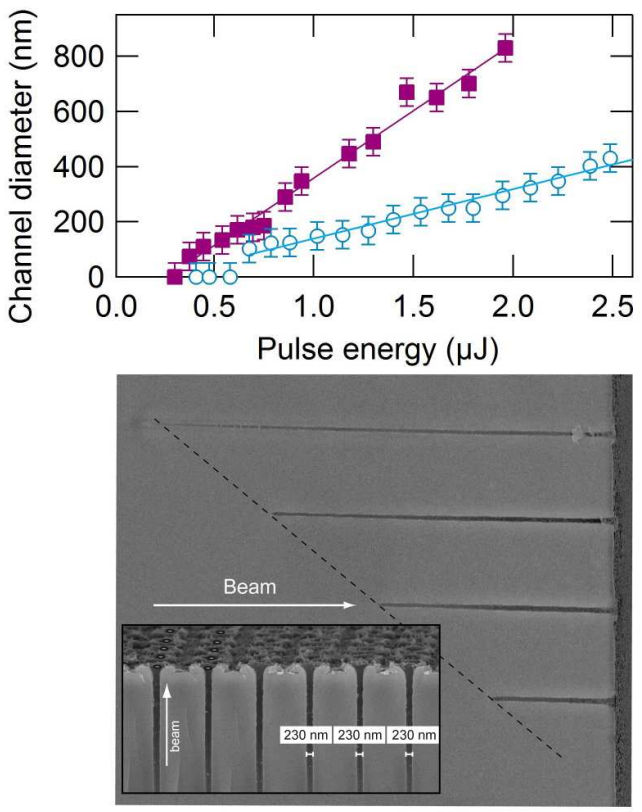

Fig. 3 (top) Evolution of channel mean diameter with pulse energy for two different conical angles: $\theta_{1}=25^{\circ}$ (full squares) and $\theta_{2}=17^{\circ}$ (open circles). (bottom) SEM image showing four machined channels processed with virtual axicon position differing by $6 \mu \mathrm{m}$ between each laser shot. (inset) Profile of a square array of identical channels showing the reproducibility of the technique.

$z<-5 \mu m$. It follows that the Bessel pulse propagation is unperturbed when the distance $D$ to the exit side is linearly reduced or increased up to a distance of $\sim 5 \mu \mathrm{m}$ after the sample entrance side. This is in accordance with our experimental results showing that a linear translation of the virtual axicon does not affect the channel diameter, but only linearly varies the channel length.

\section{Influence of previously drilled channels}

The inset of figure 3 shows the profile of a square matrix of nanochannels drilled sequentially with obvious applications to nano-photonics or nano-phononics. It first demonstrates that the nanochannel drilling process is highly reproducible. However, it seems surprising that the influence of previously drilled $N$ channels could be negligible on the propagation of the $N+1$ laser pulse. Therefore, we numerically investigated the linear propagation of a Bessel pulse in glass where a half-space is composed by a $1.6 \mu \mathrm{m}$-pitch square array of nanochannels $(0.2 \mu m$ diameter and $20 \mu m$ length). Although accurate results should be obtained by using the nonlinear model presented above, the highly non-symmetric propagation medium renders the computation very demanding. For a qualitative comparison, we therefore chose a linear propagation model based on the numerical angular spectrum of plane waves approach. 


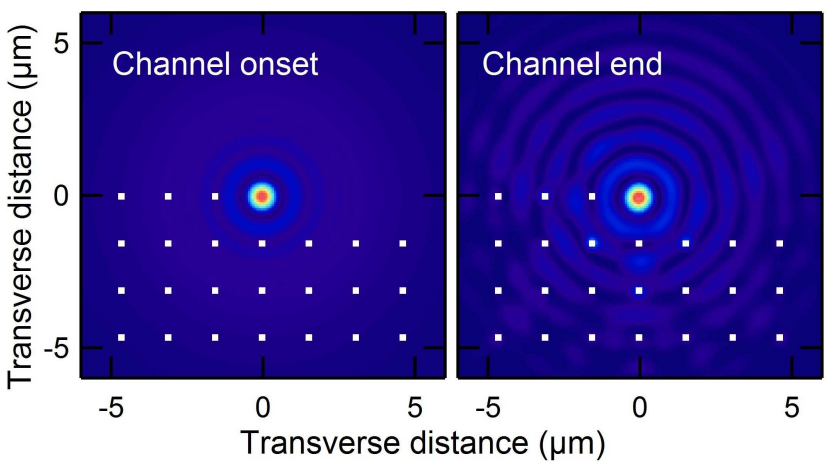

Fig. 4 Comparison of the intensity profiles at the onset (left) and at the end(right) of channel array. White dots indicate the position of previously drilled channels.

Figure 4 compares the intensity profiles at the onset of the channels $(z=5 \mu \mathrm{m})$ and at the exit side of the sample $(z=25 \mu \mathrm{m})$. The absorbers appear in white for reference. We observe that the beam profile is almost unperturbed. With a log-scale (not shown), it is clear that diffraction and absorption do occur. Nevertheless, we have shown before that plasma generation occur preferentially along the central intensity core. This demonstrates that the robustness of Bessel beams is a key benefit for processing arrays of channel with pitch greater than $\sim 1 \mu \mathrm{m}$. Below this value, a correct modelling would require to include near field effects.

\section{Conclusion}

In conclusion, we have reviewed our work applying femtosecond Bessel beams to laser ablation. Our results demonstrate the fabrication of high-aspect ratio nanochannels in glass using single shot femtosecond Bessel beams, and our simulations allow us to interpret this in terms of the stationarity of the nonlinear pulse propagation and to the robustness of Bessel beams to the presence of obstacles. We anticipate application of Bessel beams in a wide range of nanofabrication technology where high aspect structures are required.

The authors acknowledge fundings from Institut Universitaire de France, Region Franche-Comte, University of Franche-Comte, and the Agence Nationale de la Recherche contracts 2011-BS04-010-01 NANOFLAM and ANR-09-BLAN-0065 IMFINI.Computations have been performed on the supercomputer facilities of the Mesocentre de calcul de Franche-Comté.

\section{References}

1. J. Durnin, J.J. Miceli and J.H. Eberly, Phys. Rev. Lett. 58 (1987) 1499.

2. F.O. Fahrbach, P. Simon and A. Rohrbach, Nat Photon 4 (2010) 780-785.

3. V.Garcés-Chávez et al, Nature 419 (2002) 145.
4. M.A. Porras, et al, Phys. Rev. Lett. 93 (2004) 153902.

5. P. Polesana et al, Phys. Rev. A 77 (2008) 043814.

6. I. Toytman, D. Simanovski and D. Palanker, Opt. Express 18 (2010) 24688.

7. F. Courvoisier et al, Opt. Lett. 34 (2009) 3163-3165.

8. M. K. Bhuyan et al., Opt. Express 18 (2010) 566-574.

9. M. K. Bhuyan et al., Appl. Phys. Lett. 97 (2010) 081102.

10. V. Jarutis, R. Paskauskas and A. Stabinis, Opt. Commun. 184 (2000) 105-112.

11. A. Vasara, J. Turunen and A.T. Friberg, J. Opt. Soc. Am. A 6 (1989) 1748-1754.

12. T. Cizmar, K. Dholakia, Opt. Express 17 (2009) 15558.

13. Y.-Y. Yu et al, Appl. Opt. 50 (2011) 6384.

14. B. Yalizay et al, Appl. Phys. Lett. 100 (2012) 031104.

15. Y. Matsuoka, Y. Kizuka and T. Inoue, Appl. Phys. A 84 (2006) 423-430.

16. S.Juodkazis et al, Bull. Chem. Soc. Jpn. 81 (2008) 411448 .

17. A. Couairon and A. Mysyrowicz, Phys. Rep.441 (2007) 47-189.

18. S. I. Kudryashov et al, Appl. Phys. Lett. 91 (2007) 141111.

19. Y. V. White et al Opt. Express 16 (2008) 14411.

20. J. F. Herbstman and A. J. Hunt, Opt. Express 18 (2010) 16840 .

21. A. Couairon et al, Phys. Rev. B 71, 1-11 (2005).

22. A. Mermillod-Blondin et al., Phys. Rev. B 77 (2008) 1-8.

23. R. R. Gattass and E. Mazur, Nat. Photon. 2 (2008) 219225.

24. A. Couairon et al, Eur. Phys. J. Special Topics 199 (2011) 5-76.

25. L. Bergé et al, Rep. Prog. Phys. 70 (2007) 1633-1713. 